\title{
Analysis of Cardiac Rhythm using Backdated ECG Data Repository
}

\author{
Priya U. Thakare \\ Professor \\ Department of Computer Engineering, Sinhgad \\ Institute of Technology and Science, Pune-411041, \\ India \\ Saloni Vichare \\ Department of Computer Engineering, Sinhgad \\ Institute of Technology and Science, Pune-411041, \\ India
}

\author{
Nayan Raina \\ Department of Computer Engineering, Sinhgad \\ Institute of Technology and Science, Pune-411041, \\ India \\ Sharayu Kadam \\ Department of Computer Engineering, Sinhgad \\ Institute of Technology and Science, Pune-411041, \\ India
}

\begin{abstract}
Machine leaning has become an integral part of human research now a day. People are tending to select more automatic system rather than going with the manual handling. Data mining is that domain which has shown an improvement in health care industry. The proposed system deals with the classifier to study the cardiac rhythm of undiagnosed cardiac patients. However, there are many studies which were practiced over the multimedia analysis of the cardiac rhythm. The approach in this system has used the ECG dataset which will train our machine and classify the dataset. Through this dataset the following classes: Ventricular Tachycardia (VT), Ventricular Fibrillation (VF), Pulseless Electrical Activity (PEA), Asystole (AS), and Pulse-Generating Rhythm (PR) can be determined. In this dataset, features were selected using wrapper-based feature selection architecture. The ECG dataset used in this approach were practiced on various algorithms: Bayesian decision theory, k-nearest neighbor, klocal hyperplane distance nearest neighbor, artificial neural network. After classifying using these algorithms, the result of each was combined through Ensemble Decision Tree (EDT).
\end{abstract}

\section{General Terms}

Feature Selection, Classification, Data Mining, Machine Learning, Prediction

\section{Keywords}

Electrocardiogram, Ventricular Tachycardia(VT), Ventricular Fibrillation(VF), Pulseless Electrical Activity(PEA), Asystole(AS), Pulse-Generating Activity(PR), Bayesian Decision Tree, K-Nearest Neighbor(KNN), K-Local Hyperplane Distance Nearest Neighbor.

\section{INTRODUCTION}

Data Mining is a process where computations are made to discover patterns from large data sets. It is an interdisciplinary subfield of computer science, involving intersection of machine learning, artificial intelligence and database systems. Intelligent methods are applied to extract data from large data sets.The main aim of data mining is to extract meaningful information from large data sets and establish relationship to solve the problems and to predict future trends. Aside from the raw analysis step, it involves database and data management aspects, data processing model and interface considerations, complexity considerations, post-processing of discovered structures, visualization, and online updating. There are various classes of Data mining. The class which is incorporated in this system is Classification.

Classification is a data mining technique which finds out the group in which a particular data instance is related within a given dataset. Classification is nothing but predicting an outcome for a given input. It also predicts collections, categories, class labels based on the training set which can be used to classify newly available data. Different algorithms are used to find the relationship between data instances. Algorithms used for classification as ID3, K-nearest neighbor, Naïve Bayes, Artificial Neural Networks. Class Classifications are vague and do not imply order. Continuous or floating-point values would point to a numerical, rather than a categorical data set. A prediction model with a numerical target mostly uses a regression algorithm, rather than a classification algorithm.

The simplest form of classification is binary classification. In this, the target attribute has only two possible values: high credit rating or low credit rating. Multiclass targets have more than two values like low, medium, high, or unknown credit rating.

In the model training process, a classification algorithm is used for detecting relationships between the values of the predictors and that of the target. It uses different ways to detect relationships. These relationships are comprised into a model, and that model is then applied to a different data set in which the class assignments are unknown. Classification models are tested by comparing the predicted values to known target values in a set of training data. The data used for classification is divided into two data sets: training dataset, i.e. for building model and test dataset, i.e. for testing the model.

The further sections of the paper are structured as Literature survey Proposed System, Proposed Algorithms used for designing the system, Acknowledgement, Conclusion and References.

\section{LITERATURE SURVEY}

In this section of the chapter, a detailed description of the methods or approaches that are currently used in ECG prediction machines. This enables us to get the grip of the topic in technical aspects. Following are some of the methodologies that drew the attention - 
This paper describes and quantifies the physiologic responses to ALS over time for patients who are experiencing inhospital cardiac arrest, in an effort to provide an overall picture of the dynamic processes that occur before sustained ROSC is obtained or death is declared. [1]

This paper describes adaptive filter to suppress the CPR artifact from the ECG. This study shows that CPR artifacts can be suppressed from the human ECG using only the frequency of the compressions to model the artifact. [2]

In this paper, novel continuous wavelet transformation based morphology consistency evaluation algorithm is developed for the detection of disorganized VF from organized sinus rhythm (SR) without interrupting the ongoing chest compression. [3]

This paper proposes an impedance-based ventilation detection system to use during CPR. Main goal was to develop a detection system that is similar to the way human recognition is done to identify ventilations. [4]

This paper describes systematic cross-validation approach to reduce the possibility of overtraining, and it demonstrates that we are able to produce a best-in-class VF detector across a diverse set of databases. [5]

\section{PROPOSED SYSTEM}

The basic idea of this paper is to analyze the Cardiac Rhythm and to predict the issues in Cardiac Rhythm automatically and also to recommend suitable hospitals. Analysis of the patterns in rhythm may provide important information about the quality of therapy needed for the patient. This paper has detected a way which would reduce the time to analyze the Cardiac Rhythm. It would give the prediction of an issue in a class of an ECG in minimum amount of time with the help of data mining algorithms. And once the issue in the Cardiac Rhythm is known, the system would recommend suitable hospital according to the patient's need.

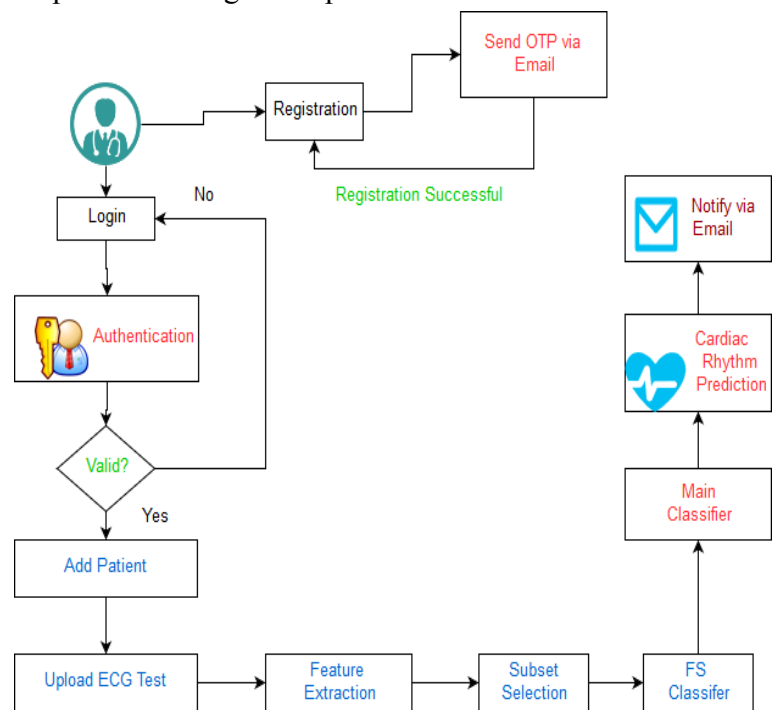

Figure1: Proposed System

Feature Extraction is an attribute reduction process. The attributes are changed after Feature extraction process. They are the combination of initial attributes. The results that we get after feature extraction process are smaller and richer sets of attributes. The models that are extracted are of high quality, as the data comprises of small and rich sets of attributes. Feature extraction converts a data set with high dimension to a one with smaller dimension. As a result it is useful for data analysis and visualization purpose, since a complex data set is easy to analyze when it is reduced to two or three dimensions.

Feature selection or Variable selection is an important part of Machine Learning and Statistics. It is the process in which selection of appropriate subset of features takes place for model building. The central idea of using a feature selection technique is that the data contains many features which are either redundant or irrelevant, and can thus be removed without losing much of information.

A feature selection algorithm is a mix of a scan method for proposing new component subsets, alongside an assessment measure which scores the distinctive element subsets. The most straightforward calculation is to test every conceivable subset of highlights finding the one which limits the blunder rate. This is a thorough hunt of the space, and is computationally recalcitrant for everything except the smallest of capabilities.

The difference between Feature extraction and subset selection is that feature extraction creates new features from functions of the original features, whereas feature selection returns a subset of the feature.

\section{PROPOSED ALGORITHMS}

\subsection{Bayesian Decision Tree}

The data preparing goal of the Bayesian is to build a probabilistic model that portrays the connections between the segments of fit arrangements in the issue space. This is accomplished by rehashing the way toward making and inspecting from a Bayesian system or likewise called as belief network that contains the contingent conditions, independencies, and restrictive probabilities between the parts of an answer. Once the system is developed, the candidate solutions are disposed of and another population of candidate solutions is created from the model. The procedure is rehashed until the point that the model joins on a fit model arrangement.

The Bayesian Optimization Algorithm is composed and examined on double string-base issues, most commonly representing binary capacity optimization problems. Bayesian systems are ordinarily constructed (developed) without any preparation with every emphasis utilizing an iterative procedure of including, expelling, and turning around links. Furthermore, past systems might be utilized as the reason for the ensuing age. A greedy hill-climbing calculation is utilized with every calculation emphasis to improve a Bayesian system to represent a population of candidate solutions.

In some problems it enables us to predict the error we will get when we generalize to novel patterns.

$$
\mathrm{P}(\mathrm{w} \mid \mathrm{X})=\mathrm{P}(\mathrm{X} \mid \mathrm{w}) \mathrm{P}
$$

Bayes formula can be expressed informally as:

Posterior $=$ likelihood $*$ prior/evidence

The pseudo code is as follows:-

Input: Bits $_{\text {num }}$, Population
size
Output: $S_{\text {best }}$

Population InitializePopulation $\left(\right.$ Bits $_{\text {num }}$, Population size $_{\text {ize }}$ )

EvaluatePopulation (Population)

$S_{\text {best }<--}$ GetBestSolution (Population, Selection ${ }_{\text {size }}$ )

While (StopCondition ()) 
Selected SelectFitSolutions (Population, Selection ${ }_{\text {size }}$ ) Model ConstructBayesianNetwork (Selected) Offspring $\square$ NULL For (i to Population size $_{\text {) }}$

Offspring $\square$ ProbabilisticallyConstructSolution (Model) End

EvaluatePopulation (Offspring)

$\mathrm{S}<--$ GetBestSolution (Offspring) Population $\square$ Combine (Population, Offspring)

\section{End}

Return $\left(S_{\text {best }}\right)$

$$
\text { End }
$$

\subsection{K-Nearest Neighbor (KNN)}

In pattern recognition, the K-Nearest Neighbor algorithm is one which has no parameters and is method used for classification and regression. In both cases, the input consists of the $\mathrm{k}$ closest training dataset in the feature space. The output depends on if $\mathrm{k}-\mathrm{NN}$ is being used for classification or regression.

In k-NN classification, the output is a member of class. An object is classified by a majority vote of its neighbors which are close it. The object is classified based on the items surrounding it and the class to which they belong. If $\mathrm{k}=1$, then the object is classified to the class of single nearest neighbor.

$\mathrm{k}-\mathrm{NN}$ is an instance-based learning, or lazy learning, where the capacity is just approximated locally and all calculation is postponed until arrangement. The k-NN calculation is among the least complex of all machine learning calculations

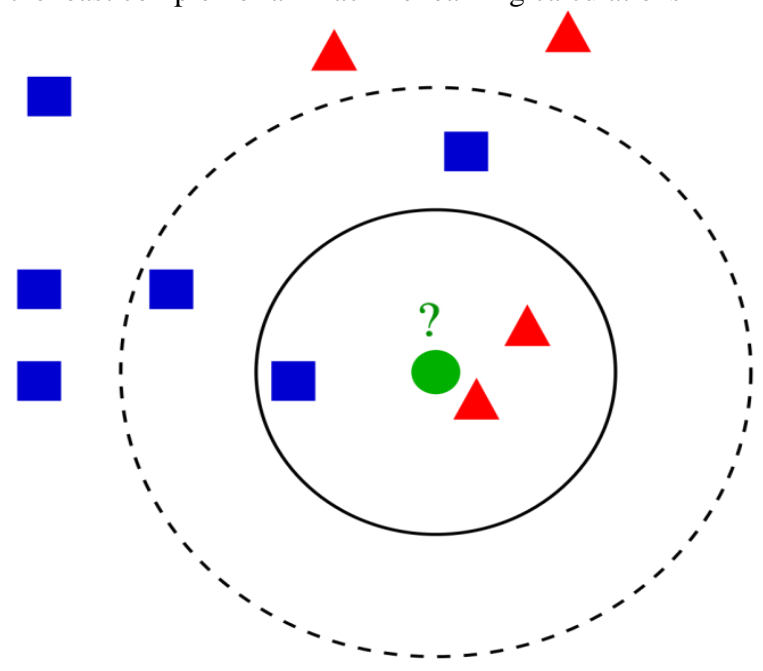

Figure 2: Example of k-NN

Example of k-NN: The test sample (green circle) is to be classified either to the first class of blue squares or to the second class of red triangles. If $\mathrm{k}=3$ (solid line circle) it is assigned to the second class because there are 2 triangles and only 1 square inside the inner circle. If $\mathrm{k}=5$ (dashed line circle) it is assigned to the first class ( 3 squares vs. 2 triangles inside the outer circle).

The training cases are vectors in a multidimensional element space, each with a class name. The training period of the calculation comprises just of putting away the vectors and class names of the training tests. In the characterization stage $\mathrm{k}$ is a client characterized consistent, and an unlabeled vector is arranged by relegating the mark which is most incessant among the $\mathrm{k}$ training tests closest to that inquiry point.

\subsection{K-Local Hyperplane Distance Nearest Neighbor (HKNN)}

HKNN is an expansion of KNN that enhances exactness to the level of portion based help vector machines. In HKNN each class is demonstrated as a low dimensional complex inserted in a high dimensional element space, and the class of an inquiry test is that of the closest class-particular complex. Class-particular manifolds are spoken to by a set of nearby hyperplanes dictated by the $\mathrm{K}$-closest focuses to an inquiry test. We utilized a regularized Euclidean separation to decide vicinity. A decay punishment term, controlled by the regularization parameter lambda was added to punish moving far from the centroid of the K-closest focuses.

\subsection{Artifical Neural Network}

Artificial neural networks (ANNs) are computing systems inspired by the biological neural networks that which are part of animal brains. Such systems learn to do tasks by considering examples, generally without task-specific programming. It is been found in most of the applications that it is difficult to express in a traditional computer algorithm using rule-based programming.

An ANN is based on a collection of connected units called artificial neurons. Each connection between neurons can transmit a signal to another neuron. The receiving neuron does then processing of the signal and sends signal to the downstream neurons connected to it. Neurons have state, which is represented by real numbers, typically between 0 and

Neurons and neurotransmitters may likewise have a weight that changes as learning continues, which can increment or abatement the quality of the signal that it sends downstream. Further, they may have an edge with the end signal that is exclusive if the total signal is underneath (or over) that level.

Typically, neurons are organized in layers.ANN consists of three different layer. Input layer, hidden layer, output layer. Different layers perform different kinds of modification on their inputs. Signals travel from input layer to output layer, possibly after traversing the layers multiple times.

The applications of neural networks are computer vision, speech recognition, social network filtering, medicine diagnosis and many more.

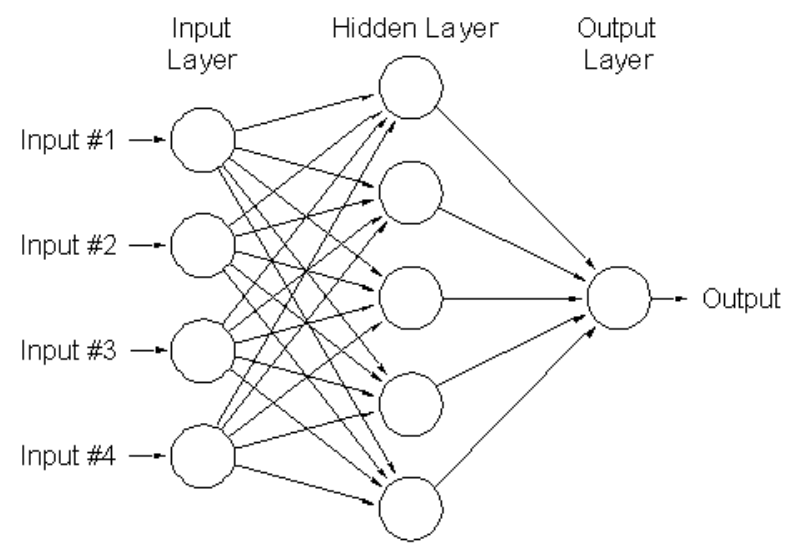

Figure 3: Three Layers of ANN

\subsection{ENSEMBLE OF DECISION TREE}

Ensemble algorithms consolidate the yields of numerous classifiers to enhance execution. We utilized ensemble decision trees (EDT), and the gathering's decent variety was 
accomplished utilizing sacking, i.e., bootstrapped copies of the training data set. Every imitation, the span of the training set, was obtained by arbitrary selection with substitution. In this way, preparing subsets note worthy cover, with many specimens display in many subsets, furthermore, a few specimens display numerous circumstances in a subset. Each training information subset was bolstered into a choice tree, which creates adequately extraordinary choice limits. The test information was ordered by taking the greater part vote of all classifiers. In this work we utilized EDTs with various number of trees $(\mathrm{NDT}=20 ; 50 ; 100 ; 200 ; 300 ; 500)$, and a root architecture (i.e., choice trees with many leaves and branch hubs).

\section{CONCLUSION}

Heart related problems are increasing day by day. In such situations time is a crucial factor. The proposed system will be significantly used in healthcare industry. It will reduce analysis time of ECG which is otherwise time consuming. After analysis of ECG and displaying in parts of heart rhythm it will also notify the patient about nearby hospitals where treatment is possible and also the cost factor.

In future, the proposed system can be made more efficient by increasing accuracy of the algorithms used. If there arises a new case which has not occurred before then, it must be updated into the database.

\section{ACKNOWLEDGEMENT}

This research was carried out under the keen guidance of Prof. Priya U. Thakare

\section{REFERENCES}

[1] Trond Trygve Eftestøl, Rune Wiseth, Benjamin S.Abella, Eirik Skogyoll, "Clinical state transitions during advanced life support (ALS) in in-hospital cardiac arrest," Elsevier, Resuscitation 84 (2013) 1238-1244.

[2] Unai Irusta, Jes'us Ruiz, Sof'1a Ruiz de Gauna, Irusta, Jes'us Ruiz, Sof'1a Ruiz de Gauna, "A least mean-square filter for the estimation of the cardiopulmonary resuscitation artifact based on the frequency of the compressions," IEEE TRANSACTIONS ON BIOMEDICAL ENGINEERING, VOL, 56, NO. 4, APRIL 2009.

[3] Yongqin Li, Joe Bisera, Max Harry Weil, Wanchun Tang, "An algorithm used for ventricular fibrillation detection without interrupting chest compression," Biomedical Engineering, IEEE TRANSACTIONS ON BIOMEDICAL ENGINEERING, VOL. 59, NO. 1, JANUARY 2012 .

[4] Martin Risdal, Sven Ole Aase, Mette Stavland, Trygve Eftestøl, "Impedance-based ventilation detection during Cardiopulmonary resuscitation," IEEE TRANSACTIONS ON BIOMEDICAL ENGINEERING, VOL. 54, NO. 12, DECEMBER 2007.

[5] Tanis Mar, Sebastian Zaunseder, Juan Pablo Mart'inez, Mariano Llamedo, and R"udiger Poll, "Optimization of ECG classification by means of feature selection," IEEE TRANSACTIONS ON BIOMEDICAL ENGINEERING, VOL. 58, NO. 8, AUGUST 2011 